\title{
miR-106b-5p modulates acute pulmonary embolism via NOR1 in pulmonary artery smooth muscle cells
}

\author{
HEMING CHEN $^{1,2}$, QIANG MA ${ }^{1}$, JUNBO ZHANG $^{1}$, YAN MENG $^{1}$, LONGFEI PAN $^{3}$ and HONGYAN TIAN ${ }^{1}$ \\ ${ }^{1}$ Department of Peripheral Vascular Medicine, First Affiliated Hospital of Xi'an Jiaotong University, Xi'an, Shaanxi 710004; \\ ${ }^{2}$ Department of Endocrinology, Ankang Central Hospital, Ankang, Shaanxi 725000; ${ }^{3}$ Department of Emergency Medicine, \\ Second Affiliated Hospital of Xi'an Jiaotong University, Xi'an, Shaanxi 710004, P.R. China
}

Received November 23, 2019; Accepted February 17, 2020

DOI: $10.3892 /$ ijmm.2020.4532

\begin{abstract}
Acute pulmonary embolism (APE) is a common cause of acute cardiovascular failure and has a high morbidity and mortality rate. Inhibiting the excessive proliferation and migration of pulmonary artery smooth muscle cells (PASMCs) is a potential treatment strategy following an APE. Various microRNAs (miRNAs/miRs) have been shown to regulate cell proliferation, apoptosis and other physiological processes. However, the specific mechanisms underlying the action of multiple miRNAs are still not understood in APE. In the present study, the role of miR-106b-5p on APE was demonstrated in platelet-derived growth factor (PDGF)-induced PASMCs in vitro and in an APE-mouse model in vivo. The results showed that miR-106b-5p expression was downregulated in PDGF-induced PASMCs and APE mice, and NOR1 levels were upregulated. Proliferating cell nuclear antigen (PCNA) expression levels in cells and proliferation of PASMCs proliferation and migration were reduced following treatment with miR-106b-5p agomiR, and increased following treatment with miR-106b-5p antagomiR. miR-106b-5p targeted the 3 ' untranslated region of NOR-1 mRNA and reduced NOR1 expression. NOR1 overexpression reversed the effects of miR-106-5p on PDGF-induced PASMCs. The functional roles of miR-106b-5p in PDGF-induced PASMCs and an APE mouse-model, and the underlying molecular mechanisms were evaluated. AgomiR-106b-5p improved APE-induced mortality and pulmonary vascular proliferation in mice. These data suggest that miR-106-5p is a novel regulator of proliferation
\end{abstract}

Correspondence to: Mr. Longfei Pan, Department of Emergency Medicine, Second Affiliated Hospital of Xi'an Jiaotong University, 157 West Fifth Road, Xi'an, Shaanxi 710004, P.R. China

E-mail: panlonf@qq.com

Professor Hongyan Tian, Department of Peripheral Vascular Medicine, First Affiliated Hospital of Xi'an Jiaotong University, 277 Yanta West Road, Xi'an, Shaanxi 710004, P.R. China

E-mail: tianhhyy@mail.xjtu.edu.cn

Key words: acute pulmonary embolism, miR-106b-5p, pulmonary artery smooth muscle cells, NOR1, proliferation of PASMCs and of pulmonary vascular remodeling through PDGF-induced PASMCs in an APE mouse model via targeting NOR1. These results expand the understanding of the pathogenesis underlying APE and highlight potential novel therapeutic targets.

\section{Introduction}

Acute pulmonary embolism (APE) is a common cause of acute cardiovascular failure and is associated with high morbidity and mortality rates $(1,2)$. The embolism causing obstruction of the pulmonary vasculature usually leads to pulmonary hypertension (PH) and cardiac failure. Studies have demonstrated that pulmonary artery smooth muscle cells (PASMCs) excessively proliferate and migrate to the pulmonary artery intima following APE, which results in reconstruction of the pulmonary vasculature and thus increases pulmonary vascular resistance $(3,4)$.

MicroRNAs (miRNAs/miRs) are 22 nucleotide-long non-coding RNAs, which serve as post-transcriptional modulators of the expression of target genes through binding to the 3' untranslated regions (UTRs) of their respective target genes. miRNAs affect mRNA expression levels through inhibiting translation or inducing mRNA degradation (5). Various miRNAs have been shown to regulate cell proliferation, death and other physiological processes. Dysregulated expression of various miRNAs is associated with several diseases, including cardiovascular diseases, such as PH. Studies have shown that abnormal expression of miRNAs contribute to the pathogenesis of hypoxia-induced $\mathrm{PH}$ (6), such as miR-31a-5p through targeting of TP53 (7), miR-135a through regulation of bone morphogenetic protein receptor type-2 (BMPR2) levels (8) and miR-17 through targeting of mitofusin-2 (9). Yue et al (10) showed that miR-143/145 promotes hypoxia-induced proliferation and migration of PASMCs, and improves hypoxia-induced $\mathrm{PH}$ through targeting ABCA1. Courboulin et al (11) demonstrated that miR-204 serves a significant role in decreasing proliferation, vascular remodeling and regulating pulmonary artery blood pressure in PH, through targeting of SHP2 (11). Several miRNAs have been identified as biomarkers for chronic thromboembolic pulmonary hypertension (CTEPH) and APE. miR-759, Let-7d, Let-7b and miR-22 have been demonstrated to modulate fibrinolysis, which contributes to 
the development of CTEPH (12). Let-7d suppresses proliferation of PASMC and Let-7b targets TGBFR1 and endothelin-1 reducing migration of pulmonary artery endothelial cells and PASMCs $(13,14)$. Recent studies have shown that expression of miR-23a, miR-221, miR-27a/b, miR-1233 and miR-28-3p are significantly increased in the plasma of patients with APE compared with healthy individuals, and may thus serve as potential biomarkers for APE (1,15-18). Zhang et al (19) demonstrated that miR-23a controls the proliferation and migration of human PASMCs by targeting BMPR2/Smad1 signaling (19). However, the specific mechanisms of several miRNAs remain to be determined in APE. To further understand the pathophysiological mechanisms underlying APE, additional studies examining the effects of miRNAs on APE required.

$\mathrm{Li}$ et al (20) demonstrated that miR-106b-5p binds to the 3'-UTR of Angiopoietin 2 (Angpt2) to induce migration and tube formation of HUVECs, and human cholesteatoma perimatrix fibroblasts (hCPFs)-exosomes transports miR-106b-5p to endothelial cells and promotes angiogenesis by upregulating expression of Angpt2 (20). miR-106b-5p is pivotal in regulating cell proliferation and migration. Thus, it was hypothesized that miR-106b-5p may be closely associated with excessive proliferation and migration of PASMCs following APE. As a member of the NR4A subfamily of nuclear receptors, NOR-1 activity is sustained at a relatively low levels in healthy vascular endothelial cells and is upregulated when affected by external stimuli $(21,22)$. NOR-1 is an effector of inflammation, growth factors, lipoproteins and thrombin, that controls the spreading, migration and proliferation of vascular cells (23-26). In the present study, miR-106b-5p was downregulated in PDGF-induced PASMCs and in an APE mouse model. Furthermore, miR-106b-5p targeted the 3' UTR of NOR-1 mRNA. The functional roles of miR-106b-5p in PDGF-induced PASMCs and in an APE mouse model were evaluated and the underlying molecular mechanisms were determined.

\section{Materials and methods}

Mouse model of APE. Male C57BL/6 mice (weighing $20 \pm 2 \mathrm{~g} ; \mathrm{n}=48$ ), were purchased from the animal center of Xi'an Jiaotong University and kept at $22 \pm 2^{\circ} \mathrm{C}$ with a relative humidity of $40-70 \%$, allowed to freely forage, with a $12-\mathrm{h}$ light/dark cycle and ad libitum access to food and water. All animal experiments were performed according to the National Institutes of Health's Guide for the Care and Use of Laboratory Animals and approved by the Institutional Animal Care and Use Committee of Xi'an Jiaotong University. The APE model was established through self-blood coagulum, as previously described (27). Briefly, $100 \mu \mathrm{l}$ blood was drawn from the tail veins of the mice. After coagulation, blood was clotted in a $37^{\circ} \mathrm{C}$ water bath for $30 \mathrm{~min}$ and diced into $1 \times 1 \mathrm{~mm}$ sections. A total of 30 autologous blood clots were injected followed by $0.4 \mathrm{ml}$ saline to establish the model. In the sham group, mice were injected with $0.4 \mathrm{ml}$ saline. Mice were, respectively, treated with $10 \mathrm{mg} / \mathrm{kg}$ agomiR-106b-5p (5'-UAAAGUGCU GACAGUGCAGAU-3') or agomiR-NC (5'-UUCUCCGAA CGUGUCACGU-3') for 3 consecutive days (Guangzhou RiboBio Co., Ltd.) through tail vein intravenous injections, beginning $15 \mathrm{~min}$ after establishment of the model. Following the operation, mice were given ad libitum access to water and food. The mortality of the mice in each group was monitored. After 7 days of treatment, mice were euthanized by carbon dioxide asphyxiation (flow rate displacing no more than $30 \%$ of the chamber volume/minute, mice were kept in carbon dioxide asphyxiation for 2-3 $\mathrm{min}$, followed by respiratory and cardiac arrest for another $1 \mathrm{~min}$ in the box), and lung tissue was obtained to analyze the lung index: Lung index = lung weight (mg)/body weight (g) x100\%; and to perform subsequent experiments.

PDGF-induced PASMCs model. Mouse PASMCs were purchased from ScienCell Research Laboratories, Inc. PASMCs were cultured in DMEM (Gibco; Thermo Fisher Scientific, Inc.) supplemented with 10\% FBS (Gibco; Thermo Fisher Scientific, Inc.). Once confluence had reached $80 \%$, PASMCs were treated with 10, 20 or $40 \mathrm{ng} / \mathrm{ml}$ PDGF (Sigma-Aldrich; Merck KGaA), and then treated with agomiR-106b-5p, antagomiR-106b-5p (5'-AUCUGCACUGUC AGCACUUUA-3'), agomiR-NC or antagomiR-NC (5'-UUC UCCGAACGUGUCACGU-3'), respectively, for $24 \mathrm{~h}$.

Transfection. NOR1 lentiviral activation particles (cat. no. sc-421926-LAC; Santa Cruz Biotechnology, Inc.) were used to overexpress NOR1. PASMCs at $80 \%$ confluence were treated with the lentiviral particles, incubated at $37^{\circ} \mathrm{C}$ for $6 \mathrm{~h}$ and subsequently the media was replaced. PASMCs were further cultured for $48 \mathrm{~h}$ before subsequent experiments were performed.

Cell proliferation assay. Proliferation of PASMCs was assessed using a Cell Counting Kit-8 (CCK8; Dojindo Molecular Technologies, Inc.), according to the manufacturer's protocol. A total of $3 \times 10^{4}$ cells/well were plated in 96-well plates. Following treatment, $10 \mu \mathrm{l}$ CCK 8 solution was added to the culture medium and the cultures were incubated for $1-3 \mathrm{~h}$ at $37^{\circ} \mathrm{C}$ with $5 \% \mathrm{CO}_{2}$. Absorbance was measured at $450 \mathrm{~nm}$ with a microplate reader (Invitrogen; Thermo Fisher Scientific, Inc.).

Transwell invasion assays. Transwell invasion assays were performed using Transwell chambers $(8 \mu \mathrm{m})$ coated with Matrigel (BD Biosciences; Becton, Dickinson and Company). In the bottom chamber, $750 \mu 1$ DMEM supplemented with $10 \%$ FBS was added and $250 \mu 1$ cell suspension at a density of $1 \times 10^{5} / \mathrm{ml}$ was added to the upper chamber without FBS. After culturing for $24 \mathrm{~h}$, the cells which had not invaded were removed and the remaining cells were fixed using $75 \%$ alcohol for $10 \mathrm{~min}$ at room temperature, stained with $0.5 \%$ crystal violet for $5 \mathrm{~min}$ at room temperature, and imaged in four randomly selected fields under an inverted optical light microscope (magnification, $x 10)$. The number of invaded cells in each group was counted.

Reverse transcription-quantitative (RT-q)PCR. Total RNA was obtained from the PASMCs of lung tissue samples and cells using TRIzol ${ }^{\circledR}$ reagent (Invitrogen; Thermo Fisher Scientific, Inc.). RT was performed in a $20 \mu 1$ mixture containing $1 \mu \mathrm{g}$ total RNA using a miRcute Plus miRNA First-Strand cDNA kit (Tiangen Biotech Co. Ltd.) or QuantScript RT kit (Tiangen 
Biotech Co. Ltd.) according to the manufacturer's protocols $\left(37.0^{\circ} \mathrm{C}\right.$ for $15 \mathrm{~min}, 95.0^{\circ} \mathrm{C}$ for $5 \mathrm{sec}$ and $4.0^{\circ} \mathrm{C}$ for $60 \mathrm{~min}$ ). qPCR experiments were performed using a miRcute Plus miRNA qPCR kit (Tiangen Biotech Co. Ltd.) or a SuperReal PreMix Plus (SYBR Green) (Tiangen Biotech Co. Ltd.) according to the manufacturer's protocols $\left(95^{\circ} \mathrm{C}\right.$ for $2 \mathrm{~min}$, followed by 35 cycles of $95^{\circ} \mathrm{C}$ for $15 \mathrm{sec}, 55^{\circ} \mathrm{C}$ for $30 \mathrm{sec}$ and $72^{\circ} \mathrm{C}$ for $10 \mathrm{sec}$; final elongation at $72^{\circ} \mathrm{C}$ for $10 \mathrm{~min}$ ). U6 and GAPDH were used as internal controls for miRNA and mRNA, respectively. The sequences of the primers used were: NOR1 forward, 5'-TGCTTTACGTCCTAGACCAG-3' and reverse, 5'-GTCTCGTCCACCTGATGTAT-3'; GAPDH forward, 5'-AACTTTGGCATTGTGGAAGG-3' and reverse, 5'-GGATGCAGGGATGATGTTCT-3'; miR-106b-5p, 5'-UAA AGUGCUGACAGUGCAGAU-3'; and U6. 5'-CAAATTCGT GAAGCGTTCCATAT-3'. Relative gene expression data were analyzed using reverse transcription-quantitative PCR and the $2^{-\Delta \Delta \mathrm{Cq}}$ method (28). The level of mRNA was determined by calculating the intensity ratio of NOR1 mRNA/GAPDH mRNA or miR-106b-5p/U6.

Western blotting. Total proteins were obtained from the PASMCs of lung tissue samples and cells using RIPA lysis buffer (Beyotime Institute of Biotechnology). Protein concentrations were quantified using a bicinchoninic acid protein kit (Invitrogen; Thermo Fisher Scientific,Inc.). Protein (40 $\mu \mathrm{g} / \mathrm{lane})$ was loaded onto $10 \%$ SDS-PAGE gels. Following SDS-PAGE and transfer to a PVDF membrane (EMD Millipore), membranes were blocked in 5\% BSA (Sigma-Aldrich; Merck $\mathrm{KGaA}$ ). Membranes were incubated with the primary antibodies at $4{ }^{\circ} \mathrm{C}$ overnight. The primary antibodies used were anti-NOR1 (cat. no. ab94507; 1:1,000; Abcam), anti-proliferating cell nuclear antigen (PCNA; cat. no. ab92552; 1:1,000; Abcam) and anti-GAPDH (cat. no. 5174S; 1:2,000; CST Biological Reagents Co., Ltd.). Subsequently, membranes were incubated with the Horseradish peroxidase, Goat Anti-Rabbit IgG secondary antibody (cat. no. A21020; 1:20,000; Abbkine Scientific Co., Ltd.) for $2 \mathrm{~h}$ at room temperature. Signals were visualized using Clarity Western ECL Substrate (Bio-Rad Laboratories, Inc.) and detected using a MiVnt image analysis system (Bio-Rad Laboratories, Inc.). Optical densities of the bands were calculated using Image Lab v5.2.1 (Bio-Rad Laboratories, Inc.).

Luciferase assays. Bioinformatics analysis using TargetScan v7.2 (http://www.targetscan.org/vert_72/), PicTar (http://www.pictar.org/), and miRanda (http://www. microrna.org/microrna/home.do) predicted that NOR-1 was a potential target gene of miR-106b-5p (29,30). Plasmids containing a portion of wide type (WT) or mutated (Mut) 3-UTR of the NOR1 gene were synthesized by Guangzhou RiboBio Co., Ltd. A total of $4 \times 10^{4}$ PASMCs/well were plated and co-transfected with $200 \mathrm{ng}$ luciferase vector (Promega Corporation) and $100 \mathrm{nM}$ agomiR-106b-5p or agomiR-NC using Lipofectamine ${ }^{\circledR} 3000$ reagent (Invitrogen; Thermo Fisher Scientific, Inc.). After 48 h, PASMCs were lysed and a Dual-Luciferase Reporter Assay (Promega Corporation) was then performed as follows: Luciferase Assay Reagent II was injected, the relative light unit (RLU) of Firefly luciferase activity was measured, Stop and Glo ${ }^{\circledR}$ reagent was injected, the
RLU of Renilla luciferase activity was measured and the level of RLU Firefly/RLU Renilla was analyzed according to the manufacturer's protocol.

RNA immunoprecipitation (RIP). RIP of miRNA ribonucleoprotein complex with anti-Argonaute 1 (Ago2; Abcam) or immunoglobulin G (IgG; Sigma-Aldrich; Merck KGaA) was performed as previously reported (17). When PASMCs reached $80 \%$ confluence, they were transfected with $100 \mathrm{nM}$ agomiR-106b-5p or agomiR-NC for $24 \mathrm{~h}$. Cell lysates were harvested using RIP buffer, incubated with magnetic beads bound with anti-Ago 2 or $\operatorname{IgG}$ antibodies. After digestion with proteinase $\mathrm{K}$, the precipitated complexes were collected and the RNA was extracted using TRIzol ${ }^{\circledR}$ reagent (Invitrogen; Thermo Fisher Scientific, Inc.). Subsequently RT-qPCR was performed to confirm the miR-106b-5p target was NOR1.

Statistical analysis. Data are presented as the mean \pm standard error of the mean of four experimental repeats. All statistical analyses were performed using GraphPad Prism version 7.0 (GraphPad Prism Software, Inc.). Differences between groups were compared using a Student's t-test or a one-way ANOVA followed by a post-hoc Tukey's test. $\mathrm{P}<0.05$ was considered to indicate a statistically significant difference.

\section{Results}

$P D G F$ reduces $m i R-106 b-5 p$ expression and increases activity of the NOR-1-PCNA signaling pathway in PASMCs. To examine the roles of miR-106b-5p on PASMCs, miR-106b-5p levels and NOR-1-PCNA pathway activity were detected following treatment with $0,10,20$ or $40 \mathrm{ng} / \mathrm{ml}$ PDGF in PASMCs. As shown in Fig. 1, PDGF significantly decreased miR-106b-5p levels, but increased NOR-1 mRNA and PCNA expression levels in a dose-dependent manner. miR-106b-5p levels were decreased 0.75 -fold, 0.4 -fold and 0.39 -fold when treated with 10, 20 and $40 \mathrm{ng} / \mathrm{ml}$ PDGF, respectively, compared with the control group (Fig. 1A). NOR-1 mRNA expression levels were increased 1.65-fold, 2.97-fold and 2.63-fold when treated with 10,20 and $40 \mathrm{ng} / \mathrm{ml}$ PDGF treatment, respectively, compared with the control group (Fig. 1B). PCNA expression levels were increased 1.45-fold, 2.07-fold and 2.33-fold when treated with 10, 20 and $40 \mathrm{ng} / \mathrm{ml}$ PDGF treatment, respectively, compared with the control group (Fig. 1C). Thus, PDGF treatment reduced miR-106b-5p expression levels and increased proliferation of PASMCs in a dose-dependent manner. For subsequent experiments, $20 \mathrm{ng} / \mathrm{ml}$ PDGF was used.

miR-106b-5p reverses $P D G F$-induced proliferation and migration of PASMCs. To determine the effect of miR-106b-5p on the proliferation and migration of PASMCs, agomiR-106b-5p and antagomiR-106b-5p were used to induce or suppress miR-106b-5p expression in PASMCs, respectively, and the levels of miR-106b-5p was assessed using RT-qPCR (Fig. S1A). CCK8 assays were used to assess cell viability. Consistent with previous results, PDGF significantly increased the viability of cells and agomiR-106b-5p inhibited the viability of cells compared with the control group $(\mathrm{P}<0.05)$. AgomiR-106b-5p reversed the PDGF-induced increase in viability in PASMCs compared with the PDGF group ( $\mathrm{P}<0.05$; Fig. 2A). PCNA 
A

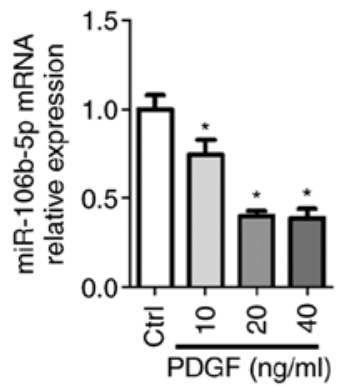

C

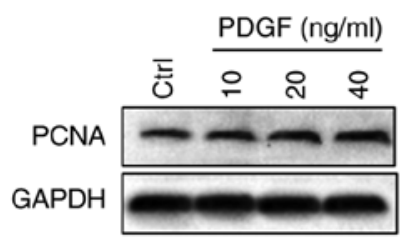

B
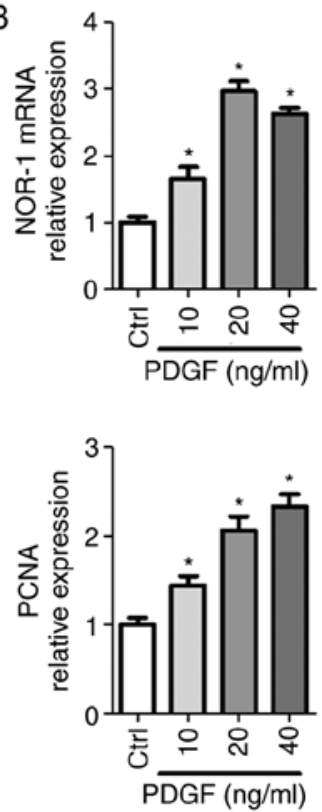

Figure 1. miR-106b-5p and NOR-1-PCNA expression levels following PDGF treatment in PASMCs. (A) miR-106-5p levels, (B) NOR1 mRNA expression levels and (C) PCNA protein expression levels following treatment of PASMCs with $0,10,20$ or $40 \mathrm{ng} / \mathrm{ml}$ PDGF for $24 \mathrm{~h} . \mathrm{n}=4$. ${ }^{*} \mathrm{P}<0.05$ vs. Ctr group. Ctrl, control; miR, microRNA; PDGF, platelet-derived growth factor; PASMC, pulmonary artery smooth muscle cell; PCNA, proliferating cell nuclear antigen.

protein expression was measured using western blotting (Fig. 2B). Protein bands and densitometry analysis showed that agomiR-106b-5p significantly abrogated the PDGF-induced increase in PCNA expression compared with the PDGF group $(\mathrm{P}<0.05)$. Cell invasion was assessed using a Transwell invasion assay (Fig. 2C). Following agomiR-106b-5p treatment, the number of invasive cells was significantly reduced, whereas PDGF increased the invasiveness of PASMCs. The number of invasive cells in the control, agomiR-NC, agomiR-106b-5p, PDGF and PDGF + agomiR-106b-5p groups were 79.33 \pm 4.84 , $77.67 \pm 5.78,56.67 \pm 4.91,128.30 \pm 6.06$ and $91.33 \pm 5.57$, respectively. Conversely, antagomiR-106b-5p inhibited miR-106b-5p expression in PASMCs, which facilitated cell proliferation (cell viability and PCNA expression) and migration compared with the control group (Fig. 3). AntagomiR-106b-5p significantly enhanced the effects of PDGF on the combined treatment group compared with PDGF alone (Fig. 3).

miR-106b-5p targets the 3'UTR of NOR-1 and reduces NOR-1 expression. Bioinformatics analysis was performed to predict the potential target genes of miR-106b-5p and NOR-1 was shown to be a potential target of miR-106b-5p (Fig. 4A). To validate this prediction, the specificity of miR-106b-5p to NOR1 mRNA was assessed using two methods: Ago2-IP and 3'UTR luciferase reporter assays. The RIP assay results demonstrated that there was an $~ 5$-fold enrichment of NOR-1 mRNA obtained from the Ago2-IP of the agomiR-106b-5p group compared with the $\mathrm{NC}$ group $(\mathrm{P}<0.05)$, suggesting that miR-106b-5pand NOR-1 were located in the same RNA-induced silencing complex (Fig. 4B). As shown in Fig. 4C, after transfection with WT-NOR-1, there was an $\sim 0.47$-fold decrease in the relative luciferase activity in the agomiR-106b-5p group compared with the NC group $(\mathrm{P}<0.05)$, and an $\sim 1.56$-fold increase in luciferase activity in the antagomiR-106b-5p group compared with the NC group $(\mathrm{P}<0.05)$. There was no difference in the luciferase activity between the groups following transfection with the Mut NOR-1 UTR (Fig. 4C). These data suggest that miR-106b-5p may target the 3'-UTR of NOR-1, which was also confirmed by the results in Fig. 4D. The mRNA expression levels of NOR-1 were significantly decreased following agomiR-106b-5p treatment and increased following treatment with antagomiR-106b-5p and/or PDGF, and agomiR-106b-5p also suppressed PDGF-induced NOR-1 expression levels $(\mathrm{P}<0.05$; Fig. 4D). Thus, these data demonstrate that miR-106b-5p negatively regulates NOR-1 expression via binding to the 3' UTR.

Activated NOR-1 reduces miR-106b-5p-inhibition of PASMC proliferation and migration. After $48 \mathrm{~h}$ transfection with NOR1 lentiviral activation particles or NC particles in PASMCs, transfection efficiency for NOR1 overexpression are shown in Fig. S1B and C. NOR1 lentiviral activation particles induced NOR1 mRNA and expression. Then cells were treated with agomiR-106b-5p and/or PDGF, and cell viability and migration were measured. NOR-1 and PDGF significantly increased cell viability and agomiR-106b-5p decreased cell viability, but the effect of agomiR-106b-5p was significantly reduced in NOR-1 activated PASMCs (Fig. 5A). Activated NOR-1 increased cell invasion in the agomiR-106b-5p treatment group, the invasive cell count was $40.7 \pm 3.84$ and $53.7 \pm 2.60$ in the agomiR-106b-5p group and the NOR-1 + agomiR-106b-5p group, respectively (Fig. 5B). Western blotting results showed that NOR-1 activation significantly increased PCNA expression in the NOR-1 + agomiR-106b-5p group compared with the agomiR-106b-5p group ( $\mathrm{P}<0.05$; Fig. 5C). Thus, NOR-1 activation reversed the inhibition of miR-106b-5p on proliferation and migration of PASMCs.

AgomiR-106b-5p improves APE-induced mortality and increases pulmonary vascular proliferation in mice. An APE mouse model was established for investigating the positive effect of miR-106b-5p in vivo. Following agomiR-106b-5p treatment, some mice were maintained for 15 days to monitor the survival and some of these mice were euthanized on day 7 to assess pulmonary vascular proliferation. Survival of the APE-induced mice after 7 days was $30 \%$ and all mice were dead after 12 days. AgomiR-106b-5p reduced the number of early deaths in APE-mice and $40 \%$ of mice were still alive at the end of the study (Fig. 6A). Considering the importance of NOR-1 and PCNA in vascular proliferation, the mRNA expression levels of NOR-1 and PCNA were assessed in the pulmonary artery. APE significantly increased the levels of NOR-1 and PCNA mRNA, whereas agomiR-106b-5p treatment significantly reduced the mRNA expression levels (both $\mathrm{P}<0.05$; Fig. 6B). As shown in the immunohistochemistry results, NOR-1 expression was higher in the narrowed blood vessels of APE-mice and lower in the neointima of pulmonary vasculature in agomiR-106b-5p-treated APE-mice (Fig. 6C). These results suggest that agomiR-106b-5p reduced APE-induced lung injury via inhibition of NOR-1 in the pulmonary vasculature of mice. 
A

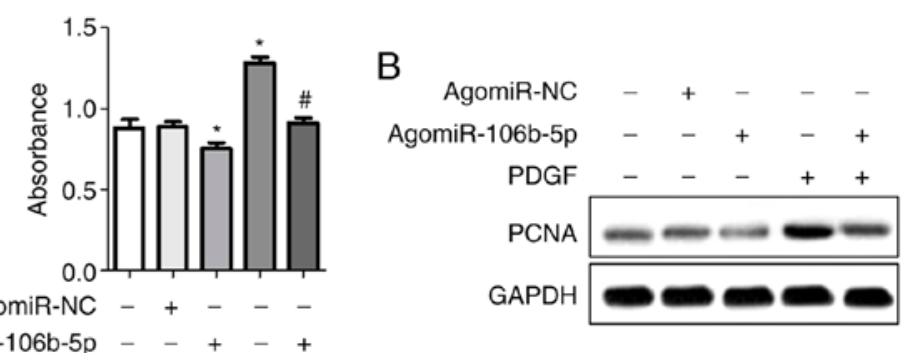

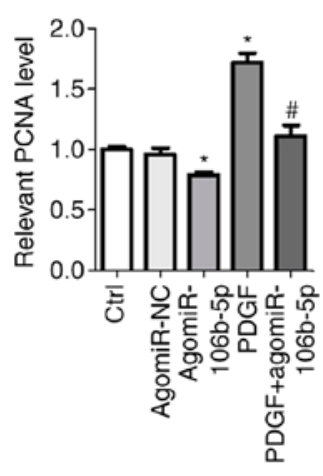

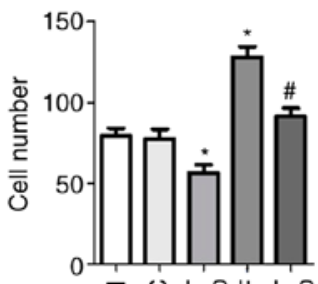

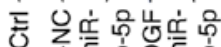
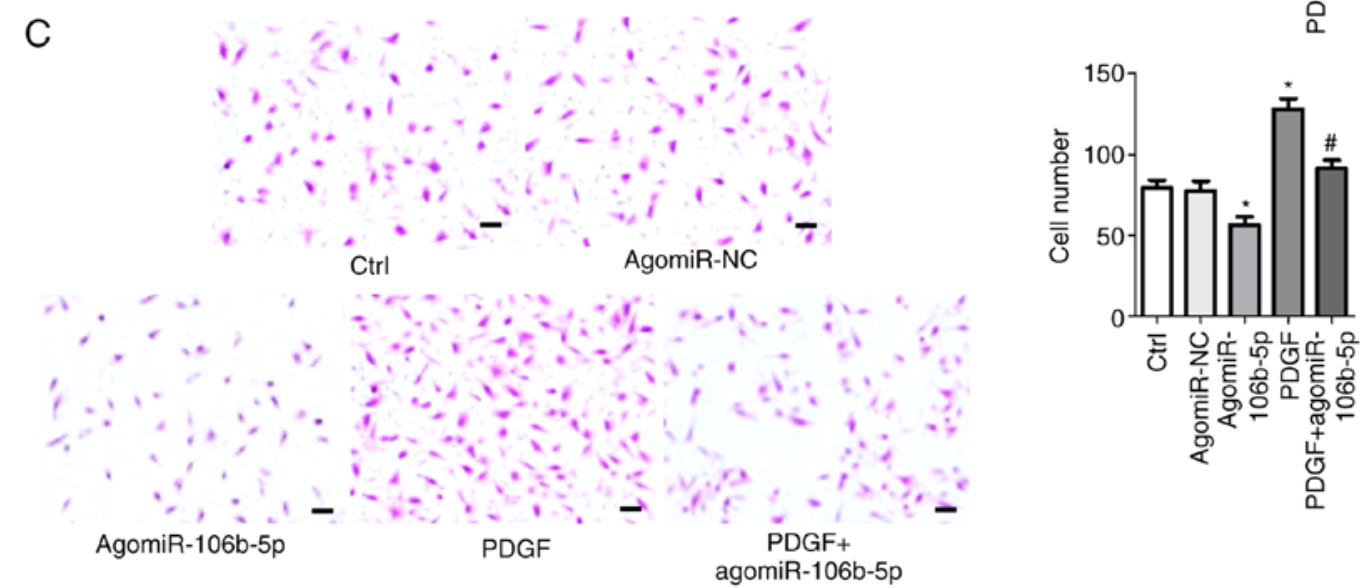

Figure 2. AgomiR-106b-5p alleviates PDGF-induced proliferation and migration of PASMCs. PASMCs were treated with $20 \mathrm{ng} / \mathrm{ml}$ PDGF and subsequently treated with agomiR-106b-5p. (A) Cell viability was assessed using a Cell Counting Kit-8 assay. (B) Western blotting and densitometry analysis of PCNA expression. (C) Transwell invasion assays were performed to assess invasion of cells following the various treatments. Scale bars: $20 \mu \mathrm{m}$. ${ }^{*} \mathrm{P}<0.05$ vs. Ctrl group. ${ }^{\#} \mathrm{P}<0.05$ vs. PDGF group. miR, microRNA; PDGF, platelet-derived growth factor; PASMC, pulmonary artery smooth muscle cell; PCNA, proliferating cell nuclear antigen; $\mathrm{NC}$, negative control; Ctrl, control.

A

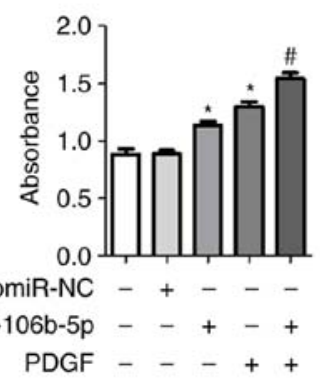

\section{B}

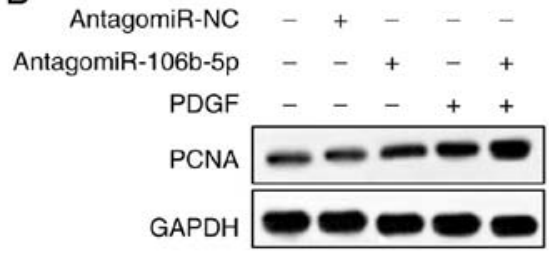

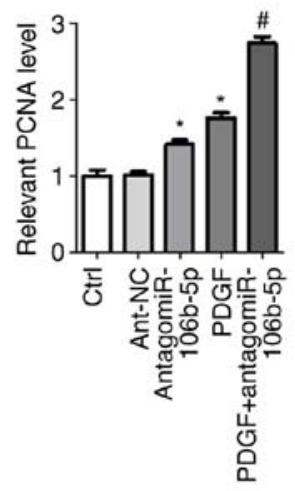

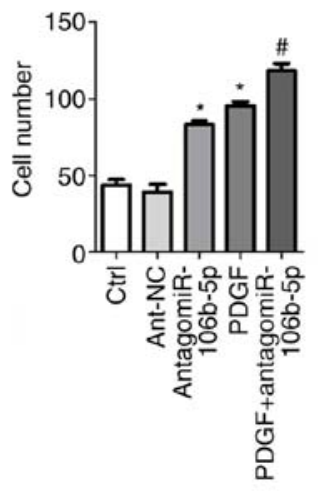

Figure 3. AntagomiR-106b-5p increases proliferation and migration of PASMCs. (A) After treatment with PDGF or antagomiR-106b-5p, the viability of PASMCs was assessed using a Cell Counting Kit- 8 assay. (B) PCNA protein expression following the various treatments. (C) Transwell invasion assays were performed to assess invasion following the various treatments. Scale bars: $20 \mu \mathrm{m}$. " $\mathrm{P}<0.05$ vs. Ctrl group. ${ }^{\sharp} \mathrm{P}<0.05$ vs. PDGF group. miR, microRNA; PDGF, platelet-derived growth factor; PASMC, pulmonary artery smooth muscle cell; PCNA, proliferating cell nuclear antigen; NC, negative control; Ctrl, control. 
A
WT NOR1 3'UTR 5'...GAAAUUGCUGACAAAGCACUUUU... IIIIIIII

mmu-miR-106b-5p 3’ UAGACGUGACAGUCGUGAAAU

MUT NOR1 3'UTR 5' ...GAAAUUGCUGACAAAGGAGUUUU...
B
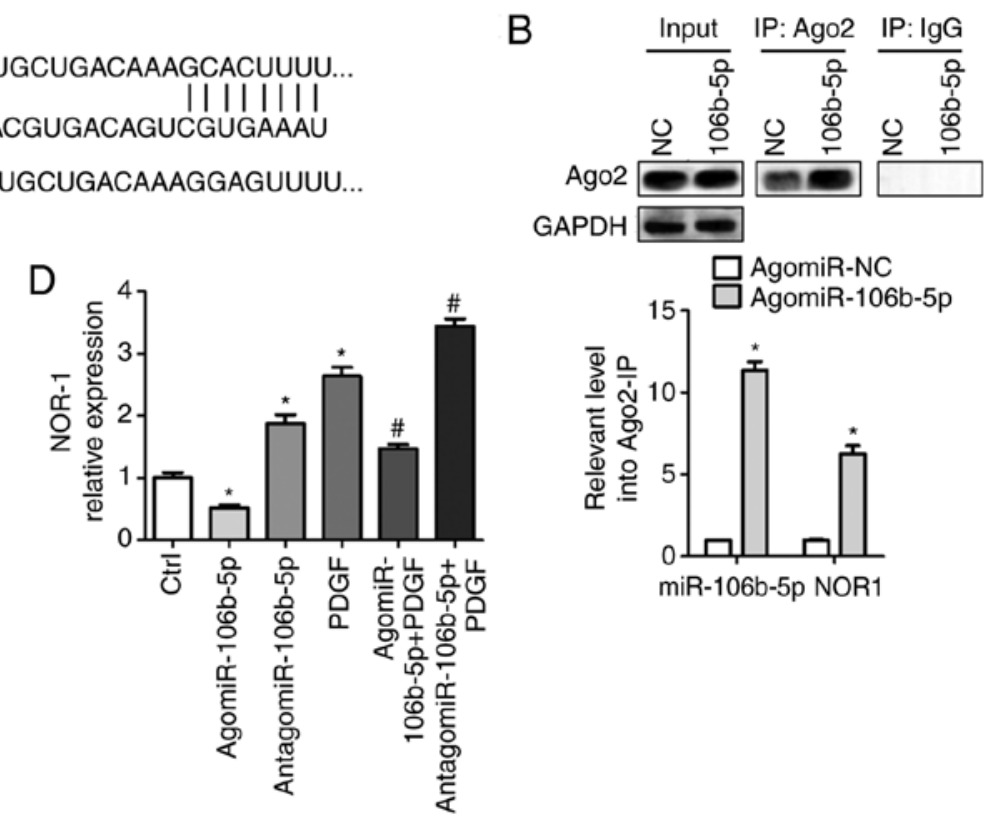

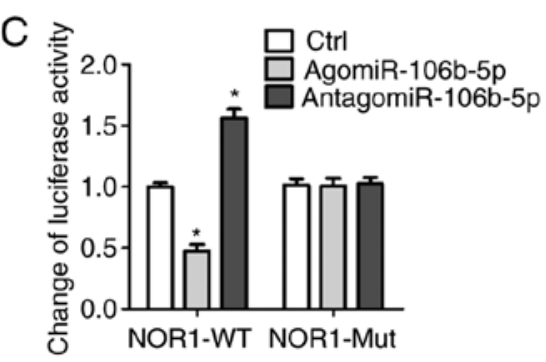

Figure 4. miR-106b-5p targeting of NOR-1 is confirmed using Ago2 immunoprecipitation and luciferase reporter assays. (A) Bioinformatics analysis using TargetScan, PicTar and miRanda predicted that NOR-1 was a potential target gene of miR-106b-5p. (B) PASMCs were transfected with 100 nM agomiR-106b-5p or agomiR-NC for $24 \mathrm{~h}$, the cells were collected and immunoprecipitated using Ago2 or IgG and the fold-changes in NOR-1 transcript levels were calculated. ${ }^{*} \mathrm{P}<0.05$ vs. agomiR-NC group. (C) After transfection with WT-NOR-1 or Mut-NOR-1 and treatment with agomiR-106b-5p or antagomiR-106b-5p in PASMCs, luciferase assays were performed to demonstrate miR-106b-5p directly targeted NOR-1. "P $<0.05$ vs. Ctrl group. (D) After treatment with agomiR-106b-5p, antagomiR-106b-5p or PDGF in PASMCs, the mRNA expression levels of NOR-1 were measured. ${ }^{\mathrm{P}}<0.05$ vs. Ctrl group. ${ }^{*} \mathrm{P}<0.05$ vs. PDGF group. miR, microRNA; PDGF, platelet-derived growth factor; PASMC, pulmonary artery smooth muscle cell; NC, negative control; WT, wild-type; Mut, mutated; Ctrl, control.
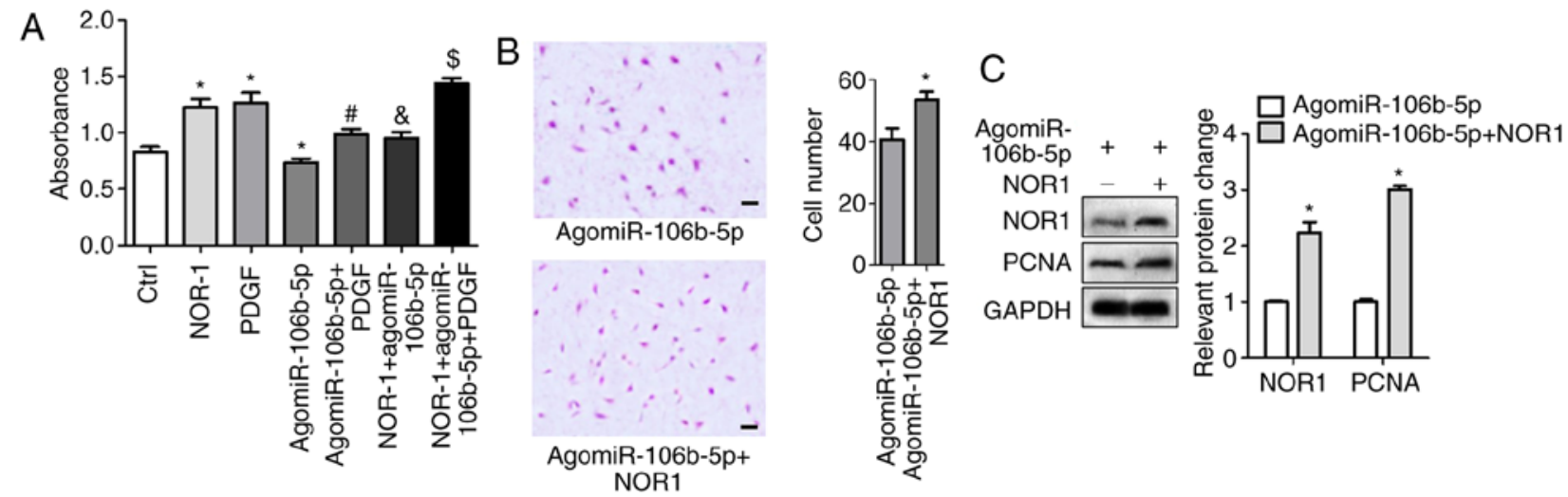

Figure 5. NOR-1 activation improves the inhibitory action of miR-106b-5p on the proliferation and migration of PASMCs. After overexpression of NOR-1 in PASMCs, cells were treated with agomiR-106b-5p and/or PDGF. (A) Cell Counting Kit-8 assays were used to assess cell viability. " $\mathrm{P}<0.05$ vs. Ctrl group. ${ }^{\#} \mathrm{P}<0.05$ vs. PDGF group. ${ }^{\&} \mathrm{P}<0.05$ vs. NOR1 group. ${ }^{\text {P }}<0.05$ vs. NOR1 + agomiR-106b-5p group. (B) Transwell invasion assays were used to assess invasion of cells. Scale bars: $20 \mu \mathrm{m}$. "P<0.05 vs. agomiR-106b-5p group. (C) NOR-1 and PCNA protein expression levels were measured using western blotting. "P< $<0.05$ vs. agomiR-106b-5p group. miR, microRNA; PDGF, platelet-derived growth factor; PASMC, pulmonary artery smooth muscle cell.

\section{Discussion}

Numerous studies have shown that the excessive proliferation and migration of PASMCs is involved in the reconstruction of pulmonary vasculature and the increase in pulmonary vascular resistance in APE $(3,4)$. In the present study, it was demonstrated that miR-106b-5p expression was downregulated in PDGF-induced PASMCs and in an APE mouse model. miR-106b-5p suppressed the proliferation and migration of PASMCs in the APE mouse model and in the PDGF-induced model, by targeting NOR-1. Furthermore,
NOR-1 overexpression reversed the reduction of proliferation and migration in miR-106b-5p agomiR-treated PASMCs. AgomiR-106b-5p improved APE-induced mortality and pulmonary vascular proliferation in mice.

As technologies have improved, a large amount of data regarding the expression of various miRNAs in APE have been obtained. Using an Affymetrix miRNA array, Miao et al (27) showed that 24 miRNAs were upregulated and 22 miRNAs were downregulated miRNAs (including miR-3148) when comparing a CTEPH group with control samples, and the number of target genes co-regulated by miR-3148 and other 
A

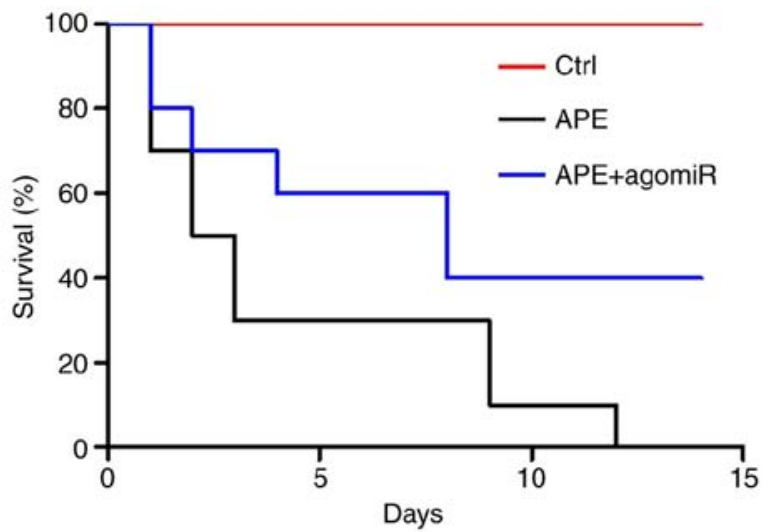

B

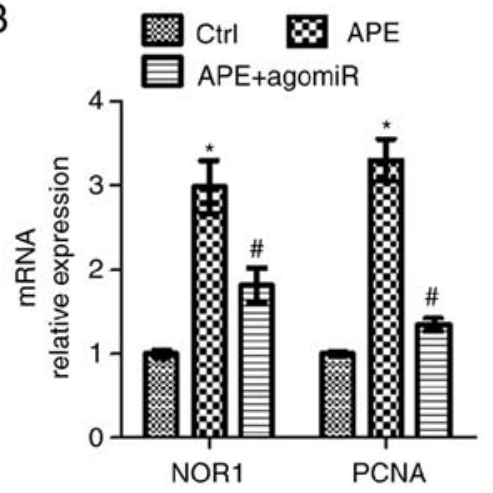

C

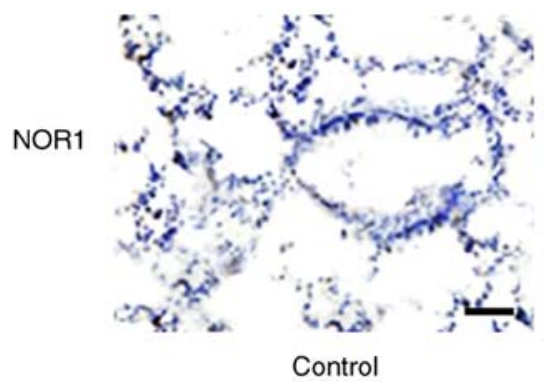

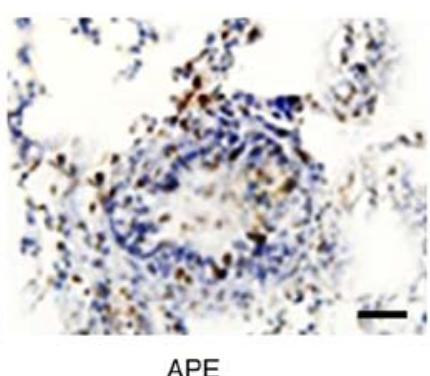

APE

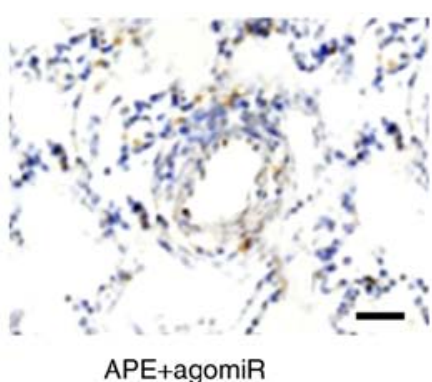

Figure 6. AgomiR-106b-5p alleviates APE-induced lung injury through inhibition of NOR-1 in a mouse model of APE. After treatment with agomiR-106b-5p in the APE mouse model, (A) survival was monitored for 15 days $(\mathrm{n}=10)$. (B) Pulmonary vascular proliferation was assessed by measuring the mRNA expression levels of NOR-1 and PCNA protein expression levels in the pulmonary artery and (C) immunohistochemistry analysis of NOR-1 was performed in the pulmonary vasculature after 7 days, $\mathrm{n}=6$. Scale bars: $20 \mu \mathrm{m} . \mathrm{n}=10$. ${ }^{*} \mathrm{P}<0.05$ vs. Ctrl; ${ }^{*} \mathrm{P}<0.05$ vs. APE group. Ctrl, control; miR, microRNA; PDGF, platelet-derived growth factor; PASMC, pulmonary artery smooth muscle cell; APE, acute pulmonary embolism.

miRNAs was the highest of all miRNAs (27). Guo et al (14) found that miR-1260, miR-602, miR-129-5p, miR-1908 and miR-483-5p were upregulated, and miR-140-3p, miR-93, miR-22, miR-106b and let-7b were downregulated in patients with CTEPH compared with the healthy controls, using a miRCURY LNA Array. As an anti-tumor factor or tumor promoter, miR-106b-5p, the matured product of miR-106b, has been reported to serve an important role in multiple types of cancer. miR-106b-5p targets CTSA to suppress the invasion and metastasis of colorectal cancer (31), but miR-106b-5p promotes stem cell-like properties of hepatocellular carcinoma cells through targeting PTEN via a PI3K/Akt signaling pathway (32). Li et al (20) demonstrated that miR-106b-5p binds the 3'-UTR of Angpt2 to induce migration and tube formation of HUVECs, and hCPFs-exosome transport to endothelial cells expressing relatively low amounts of miR-106b-5p, promoting angiogenesis through upregulation of Angpt2 (20). miR-106b-5p is pivotal in regulating cell proliferation and migration. Studies have demonstrated that PASMCs excessively proliferate and migrate to the pulmonary artery intima following APE, resulting in the reconstruction of the pulmonary vasculature and increasing pulmonary vascular resistance $(3,4)$. Thus, it was hypothesized that miR-106b-5p may be closely associated with excessive proliferation and migration of PASMCs following APE. In the present study, the expression of miR-106b-5p in patients with APE and in PDGF-induced PASMCs was determined, and was shown to be decreased compared with the control. In atherosclerotic plaques, Zhang et al (33) showed that the levels of miR-106b-5p were downregulated compared with normal vascular tissues. TNF- $\alpha$ downregulates miR-106b-5p levels and increases caspase-3 activation and cell DNA fragmentation levels, and upregulation of miR-106b-5p relieves TNF- $\alpha$-induced apoptosis through targeting PTEN in HUVECs (33). In the present study, it was shown that NOR-1 was a direct target of miR-106b-5p, PDGF treatment increased NOR-1 expression and proliferation of human PASMCs, but miR-106b-5p agomiR reduced proliferation of PASMCs through suppression of NOR-1 expression. miR-106b-5p agomiR ameliorated the thrombus-induced intimal hyperplasia and NOR-1 expression in neointimal SMCs of APE mice.

NOR-1 is an effector of inflammation, growth factors, lipoproteins and thrombin, which regulates the migration and proliferation of vascular cells (23-26). PDGF and thrombin induce rapid upregulation of NOR-1 expression, via multiple pathways, including alterations of cytosolic $\left[\mathrm{Ca}^{2+}\right]$, via the ERK-mitogen associated protein kinase signaling pathway and downstream activation of cAMP response element binding protein $(25,34)$. A previous study demonstrated that NOR1 increases $\mathrm{S}$ phase kinase-associated protein 2 expression during the proliferation which underlies neointima formation, which itself is involved in vascular proliferation (35). NOR1 also mediates the expression of several genes and proteins during vascular survival, such as cellular inhibitor of apoptosis 2, Cyclin D1, antiproteinase $\alpha-2$ macroglobulin, matrix metalloproteinase (MMP)-2 and MMP-9 (36-38). In a guidewire-induced arterial injury mouse model, NOR1 deletion reduced neointima formation during arterial injury (39), 
whereas, NOR1-overexpression in SMCs increased mitogenic activity and upregulated expression of embryonic smooth muscle myosin heavy chain, resulting in increased neointima formation in transgenic mice (40). In the present study, NOR1 expression levels were increased in PDGF-induced PASMCs and the neointimal SMCs of APE mice. PASMC viability and intimal hyperplasia were significantly reduced following administration of miR-106-5p agomiR via inhibition of NOR1. Rodríguez-Calvo et al (41) showed that the increased transcriptional activity of Cyclin D2 and increased proliferation in NOR1-overexpressing aortic SMCs and carotid artery ligation resulted in more severe neointimal formation and hemadostenosis in NOR1-transgenic mice compared with the wild-type mice. In the present study it was also shown that NOR-1 overexpression reversed the inhibition of proliferation in miR-106b-5p agomiR-treated PASMCs. Furthermore, NOR1 has been confirmed to be a downstream target of miR-638 $(42,43)$. miR-638 expression was reduced in PDGF-induced human SMCs, in a dose and time-dependent manner. NOR 1 is a downstream target gene of miR-638 and NOR1 inhibition is pivotal for miR-638-mediated suppression of PDGF-induced cyclin D1 expression and cell proliferation in human aortic SMCs (42). miR-638 also regulates aberrant proliferation and migration of airway SMCs via targeting of NOR1 and Cyclin D1, which contributes to enhanced airway smooth muscle mass associated with asthma.

In conclusion, the role of miR-106-5p in APE has not been reported previously, to the best of our knowledge and it was confirmed that miR-106-5p was a novel regulator of PASMC proliferation and pulmonary vascular remodeling via targeting of NOR1. miR-106-5p expression levels were decreased in PDGF-induced PASMCs and overexpression of miR-106-5p decreased proliferation and migration of PASMCs following PDGF stimulation. NOR1 overexpression reversed the effects of miR-106-5p on PDGF-induced PASMCs, suggesting that miR-106-5p serves a significant role in PASMCs through mediating NOR1 activity. Together, these results suggest a novel molecular mechanism underlying proliferation and migration of PASMCs, improving understanding of the pathogenesis of APE and the development of novel therapeutic targets.

\section{Acknowledgements}

Not applicable.

\section{Funding}

No funding was received.

\section{Availability of data and materials}

All data generated or analyzed during this study are included in this published article.

\section{Authors' contributions}

QM, JZ and YM performed the experiments. HC and LP were responsible for analysis and interpretation of the results. HC, LP and HT designed the experiments, and HC wrote the manuscript. All authors read and approved the final manuscript.

\section{Ethics approval and consent to participate}

All animal experiments were performed according to NIH Guide for the Care and Use of Laboratory Animals and approved by the Institutional Animal Care and Use Committee of Xi'an Jiaotong University.

\section{Patient consent for publication}

Not applicable.

\section{Competing interests}

The authors declare that they have no competing interests.

\section{References}

1. Liu T, Kang $\mathrm{J}$ and Liu F: Plasma levels of microRNA-221 (miR-221) are increased in patients with acute pulmonary embolism. Med Sci Monit 24: 8621-8626, 2018.

2. Shi Y, Zhang Z, Cai D, Kuang J, Jin S, Zhu C, Shen Y, Feng W, Ying $S$ and Wang L: Urokinase attenuates pulmonary thromboembolism in an animal model by inhibition of inflammatory response. J Immunol Res 2018: 6941368, 2018.

3. Zhou B, Sun G, Mei F and Xu H: The effects of low-molecular-weight heparin on lung and pulmonary artery injuries in acute pulmonary embolism rat model via platelet-derived growth factor- $\beta$. Saudi Pharm J 25: 564-569, 2017.

4. Xu X, Shi L, Ma X, Su H, Ma G, Wu X, Ying K and Zhang R: RhoA-Rho associated kinase signaling leads to renin-angiotensin system imbalance and angiotensin converting enzyme 2 has a protective role in acute pulmonary embolism. Thromb Res 176: 85-94, 2019.

5. Bartel DP: MicroRNAs: Genomics, biogenesis, mechanism, and function. Cell 116: 281-297, 2004.

6. Caruso P, MacLean MR, Khanin R, McClure J, Soon E, Southgate M, MacDonald RA, Greig JA, Robertson KE, Masson R, et al: Dynamic changes in lung microRNA profiles during the development of pulmonary hypertension due to chronic hypoxia and monocrotaline. Arterioscler Thromb Vasc Biol 30: 716-723, 2010.

7. Feng Q, Tian T, Liu J, Zhang L, Qi J and Lin X: Deregulation of microRNA-31a-5p is involved in the development of primary hypertension by suppressing apoptosis of pulmonary artery smooth muscle cells via targeting TP53. Int J Mol Med 42: 290-298, 2018

8. Lee HW and Park SH: Elevated microRNA-135a is associated with pulmonary arterial hypertension in experimental mouse model. Oncotarget 8: 35609-35618, 2017.

9. Lu Z, Li S, Zhao S and Fa X: Upregulated miR-17 regulates hypoxia-mediated human pulmonary artery smooth muscle cell proliferation and apoptosis by targeting mitofusin 2. Med Sci Monit 22: 3301-3308, 2016.

10. Yue Y, Zhang Z, Zhang L, Chen S, Guo Y and Hong Y: miR-143 and miR-145 promote hypoxia-induced proliferation and migration of pulmonary arterial smooth muscle cells through regulating ABCA1 expression. Cardiovasc Pathol 37: 15-25, 2018.

11. Courboulin A, Paulin R, Giguère NJ, Saksouk N, Perreault T, Meloche J, Paquet ER, Biardel S, Provencher S, Côté J, et al: Role for miR-204 in human pulmonary arterial hypertension. J Exp Med 208: 535-548, 2011.

12. Opitz I and Kirschner MB: Molecular research in chronic thromboembolic pulmonary hypertension. Int J Mol Sci 20: pii: E784, 2019.

13. Wang L, Guo LJ, Liu J, Wang W, Yuan JX, Zhao L, Wang J and Wang C: MicroRNA expression profile of pulmonary artery smooth muscle cells and the effect of let-7d in chronic thromboembolic pulmonary hypertension. Pulm Circ 3: 654-664, 2013.

14. Guo L, Yang Y, Liu J, Wang L, Li J, Wang Y, Liu Y, Gu S, Gan H, Cai J, et al: Differentially expressed plasma microRNAs and the potential regulatory function of Let-7b in chronic thromboembolic pulmonary hypertension. PLoS One 9: e101055, 2014.

15. Sarrion I, Milian L, Juan G, Ramon M, Furest I, Carda C, Cortijo Gimeno J and Mata Roig M: Role of circulating miRNAs as biomarkers in idiopathic pulmonary arterial hypertension: Possible relevance of miR-23a. Oxid Med Cell Longev 2015: 792846, 2015. 
16. Wang Q, Ma J, Jiang Z, Wu F, Ping J and Ming L: Diagnostic value of circulating microRNA-27a/b in patients with acute pulmonary embolism. Int Angiol 37: 19-25, 2018.

17. Kessler T, Erdmann J, Vilne B, Bruse P, Kurowski V, Diemert P, Schunkert H and Sager HB: Serum microRNA-1233 is a specific biomarker for diagnosing acute pulmonary embolism. J Transl Med 14: 120, 2016.

18. Zhou X, Wen W, Shan X, Qian J, Li H, Jiang T, Wang W, Cheng W, Wang F, Qi L, et al: MiR-28-3p as a potential plasma marker in diagnosis of pulmonary embolism. Thromb Res 138: 91-95, 2016.

19. Zhang Y, Peng B and Han Y: MiR-23a regulates the proliferation and migration of human pulmonary artery smooth muscle cells (HPASMCs) through targeting BMPR2/Smad1 signaling. Biomed Pharmacother 103: 1279-1286, 2018.

20. Li Y, Liang J, Hu J, Ren X and Sheng Y: Down-regulation of exosomal miR-106b-5p derived from cholesteatoma perimatrix fibroblasts promotes angiogenesis in endothelial cells by overexpression of Angiopoietin 2. Cell Biol Int 42: 1300-1310, 2018.

21. Zhao Y and Bruemmer D: NR4A orphan nuclear receptors: Transcriptional regulators of gene expression in metabolism and vascular biology. Arterioscler Thromb Vasc Biol 30: 1535-1541, 2010.

22. Martí-Pàmies I, Cañes L, Alonso J, Rodriguez C and MartinezGonzález J: The nuclear receptor NOR-1/NR4A3 regulates the multifunctional glycoprotein vitronectin in human vascular smooth muscle cells. FASEB J 31: 4588-4599, 2017.

23. Martínez-González J, Rius J, Castelló A, Cases-Langhoff C and Badimon L: Neuron-derived orphan receptor-1 (NOR-1) modulates vascular smooth muscle cell proliferation. Circ Res 92: 96-103, 2003.

24. Rius J, Martinez-González J, Crespo J and Badimon L: Involvement of neuron-derived orphan receptor-1 (NOR-1) in LDL-induced mitogenic stimulus in vascular smooth muscle cells: Role of CREB. Arterioscler Thromb Vasc Biol 24: 697-702, 2004.

25. Martorell L, Martínez-González J, Crespo J, Calvayrac O and Badimon L: Neuron-derived orphan receptor-1 (NOR-1) is induced by thrombin and mediates vascular endothelial cell growth. J Thromb Haemost 5: 1766-1773, 2007.

26. Thakar RG, Cheng Q, Patel S, Chu J, Nasir M, Liepmann D, Komvopoulos K and Li S: Cell-shape regulation of smooth muscle cell proliferation. Biophys J 96: 3423-3432, 2009.

27. Miao R, Wang Y, Wan J, Leng D, Gong J, Li J, Zhang Y, Pang W, Zhai Z and Yang Y: Microarray analysis and detection of MicroRNAs associated with chronic thromboembolic pulmonary hypertension. Biomed Res Int 2017: 8529796, 2017.

28. Livak KJ and Schmittgen TD: Analysis of relative gene expression data using real-time quantitative PCR and the 2(-Delta Delta C(T)) method. Methods 25: 402-408, 2001.

29. Liu X, Qu J, Xue W, He L, Wang J, Xi X, Liu X, Yin Y and Qu Y: Bioinformatics-based identification of potential microRNA biomarkers in frequent and non-frequent exacerbators of COPD. Int J Chron Obstruct Pulmon Dis 13: 1217-1228, 2018

30. Latonen L, Afyounian E, Jylhä A, Nättinen J, Aapola U, Annala M, Kivinummi KK, Tammela TTL, Beuerman RW, Uusitalo $\mathrm{H}$, et al: Integrative proteomics in prostate cancer uncovers robustness against genomic and transcriptomic aberrations during disease progression. Nat Commun 9: 1176, 2018.

31. Ni S, Weng W, Xu M, Wang Q, Tan C, Sun H, Wang L, Huang D Du $X$ and Sheng W: miR-106b-5p inhibits the invasion and metastasis of colorectal cancer by targeting CTSA. Onco Targets Ther 11: 3835-3845, 2018
32. Shi DM, Bian XY, Qin CD and Wu WZ: miR-106b-5p promotes stem cell-like properties of hepatocellular carcinoma cells by targeting PTEN via PI3K/Akt pathway. Onco Targets Ther 11: 571-585, 2018.

33. Zhang J, Li SF, Chen H and Song JX: MiR-106b-5p inhibits tumor necrosis factor- $\alpha$-induced apoptosis by targeting phosphatase and tensin homolog deleted on chromosome 10 in vascular endothelial cells. Chin Med J (Engl) 129: 1406-1412, 2016.

34. Nomiyama T, Nakamachi T, Gizard F, Heywood EB, Jones KL, Ohkura N, Kawamori R, Conneely OM and Bruemmer D: The NR4A orphan nuclear receptor NOR1 is induced by platelet-derived growth factor and mediates vascular smooth muscle cell proliferation. J Biol Chem 281: 33467-33476, 2006.

35. Gizard F, Zhao Y, Findeisen HM, Qing H, Cohn D, Heywood EB, Jones KL, Nomiyama T and Bruemmer D: Transcriptional regulation of S phase kinase-associated protein 2 by NR4A orphan nuclear receptor NOR1 in vascular smooth muscle cells. J Biol Chem 286: 35485-35493, 2011.

36. Alonso J, Galán M, Martí-Pàmies I, Romero JM, Camacho M, Rodríguez C and Martínez-González J: NOR-1/NR4A3 regulates the cellular inhibitor of apoptosis 2 (cIAP2) in vascular cells: Role in the survival response to hypoxic stress. Sci Rep 6: 34056 , 2016.

37. Wang CG, Lei W, Li C, Zeng DX and Huang JA: Neuron-derived orphan receptor 1 promoted human pulmonary artery smooth muscle cells proliferation. Exp Lung Res 41: 208-215, 2015.

38. Rodríguez-Calvo R, Ferrán B, Alonso J, Martí-Pàmies I, Aguiló S, Calvayrac O, Rodríguez C and Martínez-González J: NR4A receptors up-regulate the antiproteinase alpha-2 macroglobulin (A2M) and modulate MMP-2 and MMP-9 in vascular smooth muscle cells. Thromb Haemost 113: 1323-1334, 2015.

39. Nomiyama T, Zhao Y, Gizard F, Findeisen HM, Heywood EB, Jones KL, Conneely OM and Bruemmer D: Deficiency of the NR4A neuron-derived orphan receptor-1 attenuates neointima formation after vascular injury. Circulation 119: 577-586, 2009.

40. Rodríguez-Calvo R, Guadall A, Calvayrac O, Navarro MA, Alonso J, Ferrán B, de Diego A, Muniesa P, Osada J, Rodríguez C and Martínez-González J: Over-expression of neuron-derived orphan receptor-1 (NOR-1) exacerbates neointimal hyperplasia after vascular injury. Hum Mol Genet 22: 1949-1959, 2013.

41. Rodriguez-Calvo R, Guadall A, Calvayrac O, Alonso J, Ferran B, Marti I, Navarro MÁ, de Diego A, Osada J, Rodríguez C and Martínez-González J: The nuclear receptor NOR-1 regulates the activation of vascular cells and vascular remodelling in response to hemodynamic stress. Clin Investig Arterioscler 26: 66-75, 2014 (In Spanish).

42. Li P, Liu Y, Yi B, Wang G, You X, Zhao X, Summer R, Qin Y and Sun J: MicroRNA-638 is highly expressed in human vascular smooth muscle cells and inhibits PDGF-BB-induced cell proliferation and migration through targeting orphan nuclear receptor NOR1. Cardiovasc Res 99: 185-193, 2013.

43. Wang H, Yao H, Yi B, Kazama K, Liu Y, Deshpande D, Zhang J and Sun J: MicroRNA-638 inhibits human airway smooth muscle cell proliferation and migration through targeting cyclin D1 and NOR1. J Cell Physiol 234: 369-381, 2018.

This work is licensed under a Creative Commons Attribution-NonCommercial-NoDerivatives 4.0 International (CC BY-NC-ND 4.0) License. 